\title{
Children with disturbances in sensory processing: a pilot study examining the role of the parasympathetic nervous system.
}

\author{
Roseann C. Schaaf \\ Thomas Jefferson University \\ Lucy Jane Miller \\ University of Colorado Health Sciences Center \\ Duncan Seawell \\ The Children's Hospital, STAR Center, Denver, Colorado \\ Shannon O'Keefe \\ The Children's Hospital, STAR Center, Denver, Colorado
}

Follow this and additional works at: https://jdc.jefferson.edu/otfp

Part of the Occupational Therapy Commons

Let us know how access to this document benefits you

\section{Recommended Citation \\ Schaaf, Roseann C.; Miller, Lucy Jane; Seawell, Duncan; and O'Keefe, Shannon, "Children with disturbances in sensory processing: a pilot study examining the role of the parasympathetic nervous system." (2003). Department of Occupational Therapy Faculty Papers. Paper 27. https://jdc.jefferson.edu/otfp/27}

This Article is brought to you for free and open access by the Jefferson Digital Commons. The Jefferson Digital Commons is a service of Thomas Jefferson University's Center for Teaching and Learning (CTL). The Commons is a showcase for Jefferson books and journals, peer-reviewed scholarly publications, unique historical collections from the University archives, and teaching tools. The Jefferson Digital Commons allows researchers and interested readers anywhere in the world to learn about and keep up to date with Jefferson scholarship. This article has been accepted for inclusion in Department of Occupational Therapy Faculty Papers by an authorized administrator of the Jefferson Digital Commons. For more information, please contact: JeffersonDigitalCommons@jefferson.edu. 


\title{
Children With Disturbances in Sensory Processing: A Pilot Study Examining the Role of the Parasympathetic Nervous System
}

\author{
Roseann C. Schaaf, Lucy Jane Miller, \\ Duncan Seawell, Shannon O'Keefe
}

KEY WORDS

- parasympathetic nervous system

- sensory integration

- vagal tone
Roseann C. Schaaf, PhD, OTR, is Assistant Professor, Department of Occupational Therapy, Thomas Jefferson University, 130 South 9th Street, Edison 810, Philadelphia, Pennsylvania 19107; roseann.schaał@mail.tju.edu

Lucy Jane Miller, PhD, OTR, is Associate Professor, Departments of Rehabilitation Medicine and Pediatrics, University of Colorado Health Sciences Center, Denver, Colorado, and is Director, STAR (Sensory Integration Treatment and Research) Center, The Children's Hospital, Denver, Colorado.

Duncan Seawell was Research Assistant, The Children's Hospital, STAR Center, Denver, Colorado, at the time of the study.

Shannon O'Keefe, was Research Assistant, The Children's Hospital, STAR Center, Denver, Colorado, at the time of the study.
This study was a preliminary investigation of parasympathetic nervous system (PNS) functioning in children with disturbances in sensory processing. The specific aims of this study were to (1) provide preliminary data about group differences in parasympathetic functions, as measured by the vagal tone index, between children with disturbances in sensory processing and those without; (2) determine effect size and power needed for future studies; and (3) to lay the foundation for further examination of the relations of parasympathetic functioning and functional behavior in children with disturbances in sensory processing.

Participants were 15 children, nine with disturbances in sensory processing and six typically developing children. Heart period data were continuously collected for a 2-minute baseline and during administration of the 15-minute Sensory Challenge Protocol, a unique laboratory protocol designed to measure sensory reactivity (Miller, Reisman, Mclntosh, \& Simon, 2001). Groups were compared on vagal tone index, heart period, and heart rate using two-tailed, independent sample t tests.

Children with disturbances in sensory processing had significantly lower vagal tone than the typically developing sample $\left(t_{(13)}=2.4, p=.05\right)$. Statistical power analysis indicated that, for future studies, a sample size of 20 in each group would yield adequate statistical power. Although the number of subjects in this pilot study is small, the results from this study support further investigations of parasympathetic functions and functional behavior in children with disturbances in sensory processing.

Schaaf, R. C., Miller, L. J., Seawell, D., \& O'Keefe, S. (2003). Children with disturbances in sensory processing: A pilot study examining the role of the parasympathetic nervous system. American Journal of Occupational Therapy, 57, 442-449.

A pproximately $10 \%$ of the pediatric population in the United States have severe over- or underresponsiveness to sensory stimuli that interferes with daily life activities (Ahn, Miller, \& Milberger), in preparation; Baranek, Foster, \& Berkson, 1997). This atypical responsiveness has a significant impact on the quality of life for these children and their families by limiting their participation in home, school, and community activities (Cohn, Miller, \& Tickle-Degnen, 2000; Dunn, 2001; Dunn \& Westman, 1997; Miller, Reisman, McIntosh, \& Simon, 2001; Parham \& Mailloux, 1995). For example, significant food preferences due to hypersensitivities to taste and touch in the mouth may limit opportunities to participate in mealtime with family and peers. Similarly, restricted manual exploration and manipulation due to hypersensitivities may interfere with participation in school and social activities. Abnormal responsiveness to sensation may also restrict interests and activities impacting on play behavior. For example, peer interactions on the playground may be limited due to excessive fear of typical playground equipment.

Pediatric therapists, who typically receive referrals for children with these types of problems, classify this particular type of sensory processing difficulty as disturbances in sensory modulation (i.e., "a problem in the capacity to regulate and organize the degree, intensity and nature of response to sensory input in a graded and adaptive manner... [that] disrupts an individual's ability to achieve and maintain 
an optimal range of performance necessary to adapt to challenges in life") (Lane \& Miller, 2000, p. 1). For these children the ordinary sensory stimuli of daily life are so unpleasant or unnoticed as to limit their participation in daily routines and activities. Parham and Mailloux (1995) list five key limitations or disabilities commonly demonstrated by children with disturbances in sensory modulation: (1) decreased social skills and participation in play; (2) disturbances in self-confidence-self-esteem; (3) difficulties with daily life skills and at school; (4) anxiety, disturbances in attention, and disturbances in the ability to regulate reactions to others; and (5) disturbances in skill development. Parents of children with disturbances in sensory modulation report that their children have difficulty with social participation, demonstrate disturbances in self-regulation, and a lack of perceived competence and self-esteem (Cohn et al., 2000). In addition, the child's behavioral reactions to sensation or intense seeking of sensory input limit family activities and routines. For example, one parent of a child with disturbances in sensory modulation commented on the impact of the child's hyperresponsiveness on their daily life activities indicating that it restricted their ability to participate in church, movie theater outings, or shopping activities together.

Until recently, children with disturbances in sensory modulation have been studied from only a behavioral perspective using psycho-educational test results and factor analyses to identify the clusters of behavioral symptoms and categories of dysfunction (Ayres, 1965, 1979; Dunn, 1997). This approach, although valuable, provides only speculation about the potential neural mechanisms underlying disturbances in sensory modulation and does not provide data regarding the effects of behavioral symptoms on participation in activities. In order to more fully understand the child with disturbances in sensory modulation, empirical data that examines behavioral symptoms, neurological mechanisms, and their impact on participation in daily life and social activities is needed (Ayres, 1979; Lane \& Miller, 2000; Reisman \& Gross, 1992). The World Health Organization (WHO) and the National Center for Medical Rehabilitation Research (Nitkin, 2001; WHO, 2000) advocate for studies that examine limitations in functional and social participation from a multifaceted perspective including not only behavioral outcome measures of participation, but also impairment in underlying mechanisms that might contribute to restrictions in participation. The study reported here explores physiological functioning, specifically parasympathetic nervous system activity, in children with disturbances in sensory modulation and its relation to behavioral responsiveness to sensory stimuli. Inference is made regarding the potential influence of parasympathetic nervous system functioning on activity limitations and participation restrictions.

The autonomic nervous system regulates an individual's ability to adapt to environmental changes through modulation of sensory, motor, visceral, and neuroendocrine functions by means of its parasympathetic and sympathetic branches. These branches function together to promote adaptation and self-regulation in response to internal and external environmental demands. The sympathetic branch of the autonomic nervous system modulates immediate phasic responses to events, such as the fight-or-flight reaction, while the parasympathetic branch modulates the visceral and the neuroendocrine systems to maintain homeostasis and self-regulation, as well as to regulate recovery from a stressor or challenge (Nance \& Hoy, 1996). Based on the knowledge of autonomic nervous system functioning, it is logical to suspect that children with disturbances in sensory modulation, who demonstrate severe over- or underresponsiveness to sensation or inability, or both, to restore homeostasis or self-regulation after a stressor or challenge, may have disturbances in autonomic nervous system functions that influence their ability to participate in activities (McIntosh, Miller, Shyu, \& Dunn, 1999a).

To gain insight into the relations between autonomic nervous system functions and behavioral responsiveness to sensation in children with disturbances in sensory processing, Miller and her colleagues studied sympathetic nervous system functioning in children who were overresponsive to sensation using their unique laboratory protocol, the Sensory Challenge Protocol (McIntosh et al., 1999a; McIntosh, Miller, Shyu, \& Hagerman, 1999b; Miller et al., 2001; Miller et al., 1999). This protocol measures physiological activity during the administration of 50 sensory stimuli (Miller et al., 2001). Electrodermal reactivity, which is the changes in the electrical conductance of the skin as a result of eccrine sweat gland activity, is collected during the Sensory Challenge Protocol. Electrodermal reactivity is a marker for sympathetic nervous system activity (Fowles, 1986). Miller and colleagues (2001) found that children who were behaviorally overresponsive to sensory stimulation demonstrated elevated electrodermal responses and decreased habituation to sensory stimuli. Their results suggest that children with disturbances in sensory processing who are behaviorally overresponsive to sensation also show evidence of sympathetic overactivity in comparison to typically developing children. This sympathetic overactivity was found to correlate with abnormal behavioral responses as measured by the Short Sensory Profile (McIntosh et al., 1999a; Miller et al., 1999; Miller et al., 2001). Between group differences in sympathetic nervous system activity in response to sensation were also found in samples of children 
with $\mathrm{ADHD}$, autism, and fragile $\mathrm{X}$ syndrome who had disruptions in sensory modulation (Miller et al., 1999; 2001).

Miller and her colleagues studied only sympathetic functioning in children with disturbances in sensory modulation and cite the need to address the role of the parasympathetic nervous system with this population as well (Miller et al., 1999). Other literature also supports the study of parasympathetic functions in children with disabilities and suggests that parasympathetic activity may be a more valid indicator of autonomic nervous system functioning and self-regulation than sympathetic activity (Boccia \& Roberts, 2000; Fox \& Porges, 1985; Malliani, 1995; Porges, 1995). High parasympathetic activity, measured by assessing heart rate variability, has been associated with homeostasis and with the ability to adaptively cope with a wide range of changing stimuli. Decreased and disorganized parasympathetic activity is associated with a narrow range of behavioral adaptation to changing stimuli and predicts stress, vulnerability, risk status, or disturbances, or all of these, in clinical outcomes in infants and young children (DeGangi, DiPietro, Greenspan, \& Porges, 1991; DiPietro \& Porges, 1991; Malliani, 1995; Porges, 1995; Porges \& Byrne, 1992). For example, DiPietro and Porges showed that premature infants with low parasympathetic activity had poorer clinical outcomes than those with higher parasympathetic activity; and Boccia and Roberts (2000) found that boys with fragile $\mathrm{X}$ syndrome, who are extremely hyperresponsive to sensation, had decreased parasympathetic nervous system activity. Similarly, Huffman et al. (1998) showed a relation between parasympathetic activity and temperament characteristics. In this study, infants with poor behavioral adaptability also demonstrated low parasympathetic activity. Data from these studies suggest that further study of parasympathetic functioning may provide insight into the neural mechanisms underlying disturbances in sensory modulation. This information may also provide a basis for therapeutic intervention strategies that may be helpful for decreasing atypical sensory responsiveness and for measuring change in response to these interventions.

The specific aims of this pilot study are to

1) provide preliminary data regarding any group differences in parasympathetic functions between children with disturbances in sensory modulation and typically developing children;

2) use group differences to determine effect size and power needed for future studies;

3) determine if further examination of the relation of parasympathetic functioning and functional behavior in children with disturbances in sensory modulation is warranted.
In this study, we hypothesize that children with disturbances in sensory modulation, who are challenged by sensory input and have difficulty regulating their states of arousal and behavioral responsiveness, will demonstrate atypical parasympathetic functioning as measured by the cardiac vagal tone index (Porges, 1985). Specifically we hypothesize that the vagal tone of children with disturbances in sensory modulation will be lower than the vagal tone of typically developing children.

\section{Methods}

The specific aims of this study were addressed using an experimental design to evaluate the differences in parasympathetic activity between two groups of children: those with disturbances in sensory processing and a typically developing, age matched control group. Parasympathetic activity is measured using the cardiac vagal tone index (Porges, 1985). The first specific aim is accomplished by comparing the two groups on the vagal tone index. The second specific aim is accomplished by using the data from this pilot study to estimate the sample size needed for adequate power for subsequent studies. The third specific aim is accomplished by evaluating the findings to determine if further study is warranted.

Each participant was administered the Short Sensory Profile, a standardized assessment of behavioral responsiveness to sensation, and the Sensory Challenge Protocol to assess responsivity to sensation while heart data were collected for the vagal tone index.

\section{Participants}

Fifteen children, nine with disturbances in sensory modulation (mean age $=5.8, S D=2.05$ ) and six typically developing children (mean age $=6.2, S D=2.3$ ) were studied. Participants with disturbances in sensory modulation were referred from the local Children's Hospital. Inclusion criteria for the sample of children with disturbances in sensory modulation were: (1) positive clinical referral for disturbances in sensory modulation based on occupational therapy evaluation by an experienced master clinician, and a total test score of greater than $-3 S D$ from the mean on the Short Sensory Profile (McIntosh et al., 1999b); (2) no other medical diagnosis; and (3) IQ scores not greater than -1 SD from the mean (85 on the Wechsler Scales) (Wechsler, 1991). The typically developing sample was an agematched convenience sample of children of employees of the hospital. For this latter sample, a detailed developmental and educational history was completed by the parents and if no previous developmental, medical, or educational difficulties were indicated, the children were included in the 
study. Informed consent was obtained from parent or guardian prior to scheduling the participants. Table 1 provides the demographics of all children as obtained from the parents or guardians.

\section{Instrumentation}

The cardiac vagal tone index is a noninvasive marker of parasympathetic activity and is frequently cited in the literature as a marker of risk status for a variety of clinical populations and age groups (Boccia \& Roberts, 2000; DiPietro $\&$ Porges, 1991). The cardiac vagal tone index measures the variability of heart period within the frequency range of respiration. Heart period is the time interval from one peak " $r$ wave" of the electrocardiogram to the next. A specific formula developed by Porges (1985) is used to estimate parasympathetic modulation of cardiac activity from these data and is called the vagal tone index.

Data for the vagal tone index were collected using the Mini-logger Series 2000 (Mini-Mitter, 1999), a physiological data logger that monitors cardiac activity. A small belt placed on the child's chest is connected to a small batteryoperated data logger that acquires and stores heart period data from the electrocardiogram and then downloads the data for later analysis. The sensor in the Mini-logger detects heart period from the heart rate signal. Each peak of the electrocardiogram triggers the heart rate transmitter. The number of milliseconds between adjacent peaks of the electrocardiogram are counted by the logger and recorded. The data are cleaned using ML-2000, a software program that stores and displays the data.

Next, the data were transferred into MxEdit (Fox \& Porges, 1985), a software program used to edit artifacts from the data and to calculate the vagal tone index. Artifact identification and editing follows the procedures and guidelines outlined by Byrne (1993). Using MxEdit, a specific component of heart period variability called respiratory sinus arrhythmia is used to calculate only the parasympathetic influences on cardiac activity. The variance of heart period within the frequency band of respiration is extracted by applying a time-series statistic (Fox \& Porges, 1985). This method is an accepted measure of cardiac vagal activity (Berntson et al., 1997; Katona \& Jih, 1975).

The two researchers who edited the data for this study completed a detailed training and certification procedure as outlined by Byrne (1993) and were certified as reliable in artifact identification, editing and calculation of the cardiac vagal tone index by the Porges Laboratory, Department of Psychology, University of Maryland. This training involved learning the theory and procedures associated with calculation of cardiac vagal tone index, independent editing of 30 training files, and submission of 20 additional test files to
Table 1. Demographics for Age, Gender, Parent's Education and Ethnicity

\begin{tabular}{lcc}
\hline & $\begin{array}{c}\text { Children With } \\
\text { Disruptions in Sensory } \\
\text { Modulation }(n=9)\end{array}$ & $\begin{array}{c}\text { Typically } \\
\text { Developing } \\
\text { Children }(n=6)\end{array}$ \\
\hline $\begin{array}{c}\text { Age (yrs) } \\
\text { Mean }\end{array}$ & 5.78 & 6.16 \\
$\quad$ SD & 2.05 & 2.32 \\
Gender & 5 & 3 \\
$\quad$ Male & 4 & 3 \\
$\quad$ Female & & \\
Ethnicity & 9 & 4 \\
$\quad$ White/Caucasian & 0 & 2 \\
$\quad$ African American & & 2 \\
Mother's Level of Education & 0 & 4 \\
$\quad$ High School & 9 & \\
$\quad$ College & & \\
\hline
\end{tabular}

Note. $n=15$

the Porges lab for evaluation. To establish inter-rater reliability, each researcher independently edited and calculated the cardiac vagal tone index for six randomly selected files. Inter-rater reliability was calculated at $r=.96$ indicating a high level of consistency between raters.

The Sensory Challenge Protocol (SCP) is a noninvasive laboratory procedure that evaluates a child's physiologic reactivity to a series of sensory stimuli. The procedure has been used successfully in a number of studies and is described in detail in the literature (McIntosh et al., 1999a, 1999b; Miller et al., 2001). Briefly, the SCP consists of the administration of a series of 50 sensory stimuli, 10 in each of 5 sensory domains: olfactory (wintergreen oil passed under the nose), visual (a series of blinking lights), auditory (audio tape of a siren sounds), tactile (touch on the chin lightly with a feather), and vestibular (tipped back slowly while seated in a chair). The stimuli occur for 3 seconds with a pseudo-random interval of 12-17 seconds between stimuli. Sensory modalities are presented in one of two counterbalanced sequences. Cardiac activity was monitored continuously during a 2-minute baseline period and the subsequent sequence of stimuli application, which lasts approximately 15 minutes. The procedure is designed to be interesting and fun for the child and has been used successfully in a number of previous studies (McIntosh et al., 1999b; Miller et al., 1999; Miller et al., 2001). Validity and reliability estimates are not available.

\section{Procedures}

The child and family were greeted in a private waiting area and after signing the informed consent and completing the Short Sensory Profile, any questions were answered. They were then taken to the laboratory to perform the Sensory Challenge Protocol. The Sensory Challenge Protocol is designed to be interesting and fun for the child by pretending that the child is going on a "space-ship trip." The room 
is painted to resemble a space station with stars and planets painted on the walls and a mock control panel for the space ship to the child's front. There is a one-way mirror in the adjacent room so the parent can view the child during the laboratory procedure. The child steps up to a platform and sits in a child-sized chair. While the child views a preselected clip of the movie "Apollo 13," the examiner dons the small chest belt used to collect cardiac data. The movie clip shows the astronauts being equipped with their space suits and gear and is intended to mimic the experience of the child. For the study, once the child was equipped and comfortable the protocol was initiated.

Heart period data were collected using the Mini-logger series 2000 (Mini-Mitter, 1999) and were reduced and cleaned using the ML-2000 software that accompanies the Mini-logger. Data were then transferred to MxEdit (Fox \& Porges, 1985), a software program used to edit artifacts from the data and to calculate the vagal tone index.

\section{Data Analysis}

Vagal tone and other cardiac data were analyzed using twotailed, independent samples $t$ tests to compare the children with disturbances in sensory modulation to the typically developing children on the physiological measures (the cardiac vagal tone index, mean heart period, and mean heart rate). The latter two measurements were obtained during data collection for the cardiac vagal tone index and thus were included for exploratory purposes.

\section{Results}

In comparison to the typically developing participants $(n=$ $6)$, the participants with disturbances in sensory modulation $(n=9)$ had statistically significantly lower cardiac vagal tone $\left(t_{(13)}=2.4, S D=.50, p=.05\right)$ suggesting that children with disturbances in sensory modulation have less effective parasympathetic functioning than typically developing children. This finding is consistent with other studies that found low parasympathetic functions to be associated with stress vulnerability, developmental and cognitive delays, or emotional and behavioral overreactivity in infants and adults; and therefore, provides further support for the belief that parasympathetic functioning is an important factor for behavioral adaptation to stimuli (DeGangi et al., 1991; DiPietro \& Porges, 1991; Donchin, Constantine, Szold, Byrne, \& Porges, 1992; Doussard-Roosevelt, Porges, Scanlon, Alemi, \& Scanlon, 1997; Fox \& Porges, 1985).

The second finding of the current study, that the children with disturbances in sensory modulation also demonstrated lower heart period $\left(t_{(13)}=2.3, S D=265, p=.05\right)$, is consistent with the first finding. Since the cardiac vagal tone index is derived from heart period data (i.e., cardiac vagal tone index $=$ heart period variability within the frequency range of respiration) it is logical that the differences in heart period between the groups would be consistent with the difference in the cardiac vagal tone index between the groups (i.e., lower in the children with disturbances in sensory modulation in comparison to typically developing children). Alternately, the third finding, lack of significant differences in heart rate between the groups $\left(t_{(13)}=-1.5 S D\right.$ $=3.3, p>.05)$ is not surprising since heart rate is influenced by a number of factors including a combination of sympathetic and parasympathetic influences, as well as a number of other non-neural influences such as fitness level and age (Boccia \& Roberts, 2000; Cacioppo, Uchino, \& Berntson, 1994; Nance \& Hoy, 1996).

Another important finding from this study is that data was provided to estimate the power and effect size for future studies. Cohen (1977) recommends that power be at least equal to .80 with alpha set to at least .05 . In this study, comparison of children with disruptions in sensory modulation to typically developing children on the cardiac vagal tone index yielded an effect size of .90 (Mean 1-Mean 2/pooled SD) (Cohen, 1977). Based on data obtained in this project we could determine the probability that a statistical significance test at a given level ( $p=.05$ for example) would lead to rejection of a false null hypothesis in a larger study, and thus determine the sample size needed for our subsequent study. Thus, we calculated that, with an effect size of .90 and alpha set at .01 , a sample size of 20 subjects in each group is needed for future two-tailed studies (yielding an estimated power of .96) (Borenstein \& Cohen, 1988).

\section{Discussion}

The findings from this study extend the existing literature on parasympathetic functions and their relation to behavioral regulation in several ways. First this study adds to the sparse literature that investigates parasympathetic functions in individuals other than infants. As such, this study extends the utility of the vagal tone index to young children and lays the foundation for future studies of these age groups using the vagal tone index as a marker of parasympathetic activity. Second, this study demonstrates the use of the cardiac vagal tone index during a sensory challenge protocol and, thus, provides a model for future, larger, more complex studies. Third, this study is the first of its kind to examine parasympathetic activity in children with disturbances in sensory modulation. We know from previous research that these children have compromised sympathetic nervous system activity that affects their behavioral response to sensation (McIntosh, et al., 1999b). The new 
finding from this pilot study suggests that these children may be further compromised by abnormalities in their parasympathetic system and supports further research in this area. Fourth, this study lays the foundation for examining any potential links between parasympathetic nervous system functioning and activity limitations and participation restrictions in this population.

The results from this study suggest that a compromised parasympathetic system may disrupt the individual's ability to achieve and maintain a calm, focused state in the face of the sensations encountered in daily life and, by inference, affect activity participation. Our results support this idea and suggest that parasympathetic nervous system functioning may be an important factor to consider when assessing abnormal sensory responsiveness as well as more complex behaviors in children with this condition.

In addition, this study provides support for other authors who have theorized about the links between the impairment level and the participation level of the International Classifications of Functioning and Disability (WHO, 2000). For example, Dunn (1997) found that children in her study who had disturbances in sensory processing demonstrated difficulties with daily living skills that were secondary to their disruptions in sensory processing. She proposed that a person's physiological reactivity may affect sensory processing style and thus, in turn, be an important determinant of behavior (Dunn, 2001). Similarly, Way (1999), theorized that limited autonomic nervous system functioning might limit a child's intrinsic motivation for play experiences and other functional behavior. For example, an overreactive sympathetic nervous system might result in a stressful response to typical play experiences, while an underreactive parasympathetic system may not provide the support needed for physiological regulation during daily life activities. Further study of the physiological functions and their relation to activity and participation in children with disturbances in sensory modulation are needed to validate these theoretical assumptions.

Finally, the pilot data from this study demonstrate the potential utility of using the Porges (1985) method for calculation of the cardiac vagal tone index in children with disturbances in sensory modulation. Although there is ongoing discussion in the literature surrounding the use of respiratory sinus arrhythmia to index cardiac vagal tone (as used in Porges' method), the Committee on Heart Rate Variability, Society for Psychophysiological Research, recently concluded that "[the cardiac vagal tone index] is an important noninvasive window through which the complexities of the interactions between psychological states and autonomic control can be observed" (Berntson et al., 1997, p. 624). The current study, therefore, builds upon this recommendation by demonstrating strong inter-rater reliability for the use of this method to obtain cardiac vagal tone index in children with disturbances in sensory processing and provides support for future studies using similar procedures.

\section{Conclusions and Implications for Practice}

The number of subjects in this pilot study is small, and therefore results must be interpreted cautiously. This study is a first step in responding to the need noted in the literature to examine parasympathetic activity in children with disturbances in sensory modulation (McIntosh et al., 1999b). Further studies will elaborate on the potential relations between parasympathetic physiological functions and participation in everyday activities by comparing measures of participation in school, home, and community activities with parasympathetic activity measures. In addition, future studies will examine vagal reactivity during different phases of the Sensory Challenge Protocol (i.e., baseline, administration of sensation, and recovery phases) to determine if baseline parasympathetic functions as measured by the cardiac vagal tone index predict behavioral responsivity to sensation, or if baseline vagal tone alone it is a sensitive marker of reactivity. These studies will provide building blocks leading toward a larger investigation of autonomic nervous system functions and their relation to activity limitations and participation restrictions in children with disturbances in sensory modulation and will provide data that will guide occupational therapy interventions.

The finding that children with disturbances in sensory modulation may have decreased parasympathetic activity has important preliminary implications for practice. First, the importance of assisting children with disturbances in sensory modulation to establish and maintain homeostasis is evident. Adequate homeostasis (parasympathetic activity) provides a basis for the behavioral flexibility and adaptation skills needed to cope with the multiple and changing inputs in their daily environments, and thus, engage successfully in occupations. Second, the importance of intervention strategies that help maintain and regain homeostasis or self-regulation for the child, and that help the caregiver adjust his or her interaction style to facilitate this is supported. Adequate behavioral self-regulation will likely provide a foundation for higher-level skills needed for participation in functional and social activities.

\section{Acknowledgments}

We would like to thank the children and families for participating in the study, the therapists at the Denver 
Children's Hospital for their referral of children for the study, and the Wallace Research Foundation, which provided primary support for this research. Support was also obtained from NIH Grant (1 K01 HD01183-01 and 1R21 HD/AR41614-01) and the Children's Hospital Research Scholar Award, Denver, Colorado. In addition, we thank Margarette Shelton, PhD, OTR, for her editorial assistance, and Jill Jantos and Judy Benzel for their administrative support. We thank Dr. D. Matthews and other staff and faculty at The Children's Hospital, Department of Rehabilitation.

This study was supported, in part, by a grant from the National Institute of Disabilities and Rehabilitation Research (\#H133F01002). Parts of this paper were presented at the American Occupational Therapy Association Annual Conference \& Expo, Miami Beach, Florida, May 5 , 2002.

\section{References}

Ahn, R. R., Miller, L. J., \& Milberger, S. (2002). Prevalence of sensory processing impairments among kindergarten children. Manuscript submitted for publication.

Ayres, A. J. (1965). Patterns of perceptual-motor dysfunction in children: A factor analytic study. Perceptual and Motor Skills, 20, 335-368.

Ayres, A. J. (1979). Sensory integration and the child. Los Angeles: Western Psychological Services.

Baranek, G. T., Foster, L. G., \& Berkson, G. (1997). Sensory defensiveness in persons with developmental disabilities. Occupational Therapy Journal of Research, 17(3), 173-185.

Berntson, G. G., Bigger, J. T., Eckberg, D. L., Grossman, P., Kaufman, P. G., Malik, M., et al. (1997). Heart rate variability: Origins, methods, and interpretive caveats. Psychophysiology, 34, 623-648.

Boccia, M., \& Roberts, J. E. (2000). Behavior and autonomic nervous system function assessed via heart period measures: Case of hyperarousal in boys with fragile $\mathrm{X}$ syndrome. Behavior Research Methods, Instruments, and Computers, 32, 5-10.

Borenstein, M., \& Cohen, J. (1988). Statistical power analysis: A computer program. Hillsdale, NJ: Erlbaum.

Byrne, E. A. (1993). Inter-beat interval editing for heart rate variability analysis: An integrated training program with standards for student reliability assessment. Developmental Assessment Library. College Park, MD: University of Maryland at College Park.

Cacioppo, J. T., Uchino, B. N., \& Berntson, G. G. (1994). Individual differences in the autonomic origins of heart rate reactivity: The psychometrics of respiratory sinus arrhythmia and preejection period. Psychophysiology, 31, 412-419.

Cohen, J. (1977). Statistical power analysis for the behavioral sciences (2nd ed.). New York: Academic Press.

Cohn, E., Miller, L. J., \& Tickle-Degnen, L. (2000). Prenatal hopes for therapy outcomes: Children with sensory modula- tion disorders. American Journal of Occupational Therapy, 54(1), 6-12.

DeGangi, G. A., DiPietro, J. A., Greenspan, S. I., \& Porges, S. W. (1991). Psychophysiological characteristics of the regulatory disordered infant. Infant Behavior and Development, 14, $37-50$.

DiPietro, J. A., \& Porges, S. W. (1991). Vagal responsiveness to gavage feeding as an index of preterm status. Pediatric Research, 29, 231-236.

Donchin, Y., Constantine, S., Szold, A., Byrne, E. A., \& Porges, S. W. (1992). Cardiac vagal tone predicts outcome in neurosurgical patients. Critical Care Medicine, 20, 942-949.

Doussard-Roosevelt, J. A., Porges, S. W., Scanlon, J. W., Alemi, B., \& Scanlon, K. B. (1997). Vagal regulation of heart rate in prediction of developmental outcome for very low birth weight infants. Child Development, 68, 173-176.

Dunn, W. (1997). The impact of sensory processing abilities on the daily lives of young children and their families: A conceptual model. Infants and Young Children, 9, 23-35.

Dunn, W. (2001). The sensations of everyday life: Empirical, theoretical, and pragmatic considerations. American Journal of Occupational Therapy, 55, 608-620.

Dunn, W., \& Westman, K. (1997). The sensory profile: The performance of a national sample of children without disabilities. American Journal of Occupational Therapy, 51, 25-34.

Fowles, D. C. (1986). The endocrine system and electrodermal activity. In M. G. H. Coles, E. Donchin, \& S. W. Porges (Eds.), Psychophysiology: Systems, processes and applications (pp. 51-96). New York: Guilford Press.

Fox, N. A., \& Porges, S. W. (1985). The relation between neonatal heart period patterns and developmental outcome. Child Development, 56(1), 28-37.

Huffman, L. C., Bryan, Y. E., del Carmen, R., Pedersen, F. A., Doussard-Roosevelt, J. A., \& Porges, S. W. (1998). Infant temperament and cardiac vagal tone: Assessments at twelve weeks of age. Child Development, 69, 624-635.

Katona, P. G., \& Jih, F. (1975). Respiratory sinus arrhythmia: Non-invasive measure of parasympathetic cardiac control. Journal of Applied Physiology, 39, 801-805.

Lane, S. J., \& Miller, L. J. (2000). Toward a consensus in terminology in sensory integration: Part 1: Taxonomy of neurophysiological processes. Sensory Integration Special Interest Section Quarterly, 23(1), 1-4.

Malliani, A. (1995). Association of heart rate variability components with physiological regulatory mechanisms. In $\mathrm{M}$. Malik \& A. J. Camm (Eds.), Heart rate variability (pp. 202-242). Armonk, NY: Futura Publishing Company Inc.

McIntosh, D. N., Miller, L. J., Shyu, V., \& Dunn, W. (1999a). Overview of the short sensory profile (SSP). In W. Dunn (Ed.), The sensory profile: Examiner's manual (pp. 59-73). San Antonio, TX: The Psychological Corporation.

McIntosh, D. N., Miller, L. J., Shyu, V., \& Hagerman, R. J. (1999b). Sensory modulation disruption, electrodermal responses, and functional behaviors. Developmental Medicine and Child Neurology, 41, 608-615.

Miller, L. J., McIntosh, D. N., McGrath, J., Shyu, V., Lampe, M., Taylor, A. K., et al. (1999). Electrodermal responses to sensory stimuli in individuals with fragile $\mathrm{X}$ syndrome. American Journal of Medical Genetics, 83, 268-279. 
Miller, L. J., Reisman, J., McIntosh, D. N., \& Simon, J. (2001). An ecological model of sensory modulation. In S. SmithRoley, E. Imperatore-Blanche, \& R. C. Schaaf (Eds.), The nature of sensory integration with diverse populations (pp. 57-88). San Antonio, TX: The Psychological Corporation.

Mini-Mitter Company, Inc. (1999). Mini-logger series 2000. Sunriver, OR: Author.

Nance, P. W., \& Hoy, C. S. (1996). Assessment of the autonomic nervous system. Physical Medicine \& Rehabilitation, 10, 15-35.

Nitkin, R. (2001, December). Introduction to NIH \& NCMRR research support mechanisms. National institute of child health and human development training workshop. Conducted at the National Center for Medical Rehabilitation Research, Rockville, MD.

Parham, D. P., \& Mailloux, Z. (1995). Sensory integration. In J. Case-Smith (Ed.), Occupational therapy for children (pp. 329-381). St. Louis, MO: Mosby.

Porges, S. W. (1985). Method and apparatus for evaluating rhyth- mic oscillations in aperiodic physiological response systems. US Patent No 4520944.

Porges, S. W. (1995). Cardiac vagal tone: A physiological index of stress. Neuroscience and Biobehavioral Reviews, 19, 225-233.

Porges, S. W., \& Byrne, E. A. (1992). Research methods for measurement of heart rate and respiration. Biological Psychology, 34, 93-130.

Reisman, J., \& Gross, A. (1992). Psychophysiological measurement of treatment in an adult with sensory defensiveness. Canadian Journal of Occupational Therapy, 59, 248-257.

Way, M. (1999). Parasympathetic and sympathetic influences in neuro-occupation pertaining to play. Occupational Therapy in Health Care, 12, 71-86.

Wechsler, D. (1991). Wechsler intelligence scale for children (3rd ed.). San Antonio, TX: The Psychological Corporation.

World Health Organization. (2000). International classification of impairments, activities and participation: A manual of dimensions of disablement and functioning. Geneva, Switzerland: Author.

\section{Save the Date!}

Wednesday, September 24

AOTA's Second Annual

Nationa 1 School

Backpack Awareness

Day

is September 24, 2003.

Educators, teach your students how to hold a community event. To view the Community

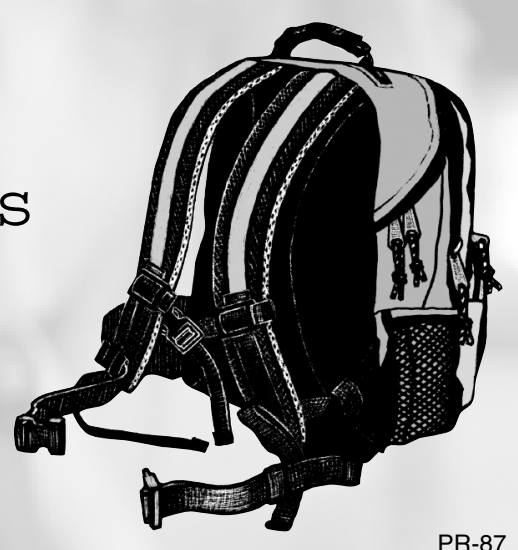

